\title{
ECONOMÍAS EXTERNAS DE RED \\ EN EL SISTEMA DE CIUDADES DEL EJE CAFETERO, 1996-2014*
}

\author{
Recibido: 28 de mayo de 2018 - Aprobado: 03 de septiembre de 2018 \\ https://doi.org/10.22395/seec.v21n48a4 \\ Mario Alberto Gaviria Ríos ${ }^{* *}$
}

\section{RESUMEN}

De manera tradicional la geografía económica ha asociado las economías de aglomeración a la concentración de actividades y población en una ciudad. Este trabajo se propuso evidenciar la generación de esas economías en la escala regional, teniendo como unidad espacial de análisis el sistema de ciudades del Eje Cafetero (Colombia) y apoyándose en un ejercicio de panel de efectos fijos. A través del ejercicio se observó la presencia de las llamadas economías externas de red en el sistema urbano estudiado, de lo que deriva que las redes hacen posible que las ciudades pequeñas puedan disfrutar de las ventajas de la aglomeración.

\section{PALABRAS CLAVE}

Externalidades; economía urbana; economía regional

\section{CLASIFICACIÓN JEL}

H23, P25, R10

\section{CONTENIDO}

Introducción; 1. Las economías externas de aglomeración: aspectos teóricos; 2. Los estudios relacionados con las externalidades de red; 3 . Aspectos metodológicos; 4. Economías externas de red en la ciudad región; 5. Conclusiones; Bibliografía.

\footnotetext{
Artículo de investigación, grupo Crecimiento Económico y Desarrollo, categoría C de Colciencias. Derivado del proyecto Redes de ciudades y configuración territorial, el caso de la formación espacial ciudad región Eje Cafetero adelantado en el Doctorado en Estudios Territoriales de la Universidad de Caldas y desarrollado entre 2016 y 2017.

* Economista, Universidad de Antioquia, Medellín. Magíster en Ciencias Económicas, Universidad Nacional de Colombia, Medellín. Profesor de la Universidad Católica de Pereira. Dirección: Calle 72 No 38A-10 Pereira. Teléfono: Teléfono fijo: (+57) (6) 3459138. Correo electrónico: mario.gaviria@ucp.edu.co
} 


\title{
EXTERNAL NETWORK ECONOMIES IN THE CITY SYSTEM OF THE COLOMBIAN COFFEE GROWING AXIS (EFE CAFETERO), 1996-2014
}

\begin{abstract}
Traditionally, economic geography has associated agglomeration economies with the concentration of activities and population in cities. This paper aims at demonstrating the generation of these economies in a regional scale, choosing the city system of the Colombian coffee growing axis as spatial unit of analysis, and relying on a fixed effects panel exercise. Throughout the exercise, the presence of so-called external network economies was observed in the studied urban system. Therefore, networks make it possible for small cities to enjoy the advantages of agglomeration.
\end{abstract}

\section{KEYWORDS}

Externalities; urban economy; regional economy

\section{JEL CLASSIFICATION}

H23, P25, R10

\section{CONTENT}

Introduction; 1. The external agglomeration economies: theoretical aspects; 2. Studies related to network externalities; 3 . Methodological aspects; 4 . External network economies in the city region; 5. Conclusions; Bibliography.

\section{ECONOMIAS EXTERNAS DE REDE NO SISTEMA DE CIDADES DO EIXO CAFEEIRO, 1996-2014}

\section{RESUMO}

De maneira tradicional, a geografia econômica tem associado as economias de aglomeração com a concentração de atividades e de população numa cidade. Este trabalho propõe evidenciar a geração dessas economias na escala regional, considerando como unidade espacial de análise o sistema de cidades do Eixo Cafeeiro (Eje Cafetero, Colômbia) e apoiando-se num exercício de painel de efeitos fixos. Por meio do exercício, foi observada a presença das chamadas "economias externas de rede" no sistema urbano estudado, do que deriva que as redes tornam possível que as cidades pequenas possam usufruir das vantagens da aglomeração.

\section{PALAVRAS-CHAVE}

Externalidades; economia urbana; economia regional.

\section{CLASSIFICAÇÃ̃ JEL}

H23, P25, R10

\section{CONTEÚDO}

Introdução; 1. As economias externas de aglomeração: aspectos teóricos; 2. Os estudos relacionados com as externalidades de rede; 3 . Aspectos metodológicos; 4 . Economias externas de rede na cidade região; 5. Conclusões; Bibliografia. 


\section{INTRODUCCIÓN}

Desde que Alfred Marshall describió el proceso de concentración localizada de empresas especializadas como generador de economías externas, la economía ha avanzado en forma significativa en la conceptualización de los procesos creadores de rendimientos crecientes y externalidades económicas, asociados a dinámicas de aglomeración espacial. Como parte de esos desarrollos, desde el paradigma de redes de ciudades se destaca la generación de estas ventajas de aglomeración en la escala regional, derivadas de la interacción entre agentes económicos que no requieren estar concentrados en una misma ciudad. En esa línea, se explora la presencia de externalidades en el sistema de ciudades de la ciudad-región Eje Cafetero, del centro occidente colombiano. Para ello se recurre a un ejercicio de panel de efectos fijos y se asume que el crecimiento de una ciudad está influido por la dinámica económica de las otras ciudades de su sistema. El ejercicio desarrollado arrojó evidencia en favor de la hipótesis de presencia de externalidades espacialmente dinámicas en ese sistema urbano, derivadas de la interacción en red de los municipios que la integran, por lo que las relaciones en red hacen posible que las ciudades pequeñas también disfruten de las ventajas de la aglomeración.

El informe se desarrolla en cinco apartados. En el primero se repasan los aspectos teóricos de las economías externas y se diferencian las visiones que las refieren a la firma (Marshall, 2005' Oughton y Whitam, 1997), al sector económico (Marshall, 2005; Hoover, 1937; Glaeser et al. 1992) y a la ciudad (Capello, 2000; Soja, 2008; Parr, 2002); en el segundo, se revisan los trabajos que han estudiado las externalidades de red; en el tercero, se presentan los aspectos metodológicos que orientaron la investigación, luego se muestran los resultados del ejercicio de estimación de las economía de red en la ciudad-región del Eje Cafetero y se finaliza con unos comentarios respecto de la temática estudiada.

\section{LAS ECONOMIIAS EXTERNAS DE AGLOMERACIÓN, ASPECTOS TEÓRICOS}

Las economías de aglomeración son una forma de externalidad que hace referencia a ventajas asociadas con el tamaño y la concentración de la población y la actividad económica en una ciudad. Los beneficios de tal concentración varían para los distintos agentes que operan en ella y es posible agruparlos en economías de consumo y economías de negocios. Las primeras hacen referencia a las ventajas que obtienen los consumidores y que se derivan de la disponibilidad de una oferta amplia y variable de servicios y productos; las segundas destacan las ventajas que

${ }^{1}$ El trabajo de Alfred Marshall Principies of economics fue publicado originalmente en 1890, el texto revisado corresponde a una edición en español de 2005. 
obtienen de esa concentración los establecimientos productivos, estas últimas son el objeto de discusión de esta parte.

El concepto de economías externas fue introducido por Marshall (2005) a partir de sus trabajos sobre la firma representativa y la noción de costes constantes. Marshall observó reducciones en los costes que no eran resultado de las decisiones de la firma y derivaban de factores como la expansión de los mercados, el acceso a un mercado amplio de mano de obra o de los mejores niveles de salud, educación y cultura provistos por otras firmas o la industria como un todo. De esa manera, el alcance primitivo del concepto se refiere a efectos que son externos a la organización, pero muchas veces internos de la industria. Marshall ilustró su idea, al analizar las ventajas derivadas de producir en una zona industrial que denominó distrito industrial, para lo cual tomó como referencia básica la concentración de la metalurgia en Sheffield (Inglaterra), formada por pequeñas y medianas empresas dedicadas de manera predominante a la misma actividad y con elevados niveles de competitividad. En ese sentido, desde su nacimiento, el concepto de economías externas ha estado muy unido a la realidad de la concentración espacial de la actividad económica.

Marshall argüía así tres razones por las que a un productor le podría resultar ventajoso situarse cerca de otros productores de su industria. En primer lugar, la concentración espacial de empresas de ese sector favorece el establecimiento de otras actividades complementarias proveedoras de inputs, servicios de carácter especializado y maquinaria específica. Además, la división del trabajo que se puede alcanzar gracias a la existencia de una masa crítica mínima de un tipo de actividad, reduce los costes de producción para el conjunto de las empresas.

En segundo lugar, una aglomeración de empresas que contrata trabajadores de similar condición, propicia la formación de un mercado de trabajo especializado, compartido por todas las empresas localizadas en ese espacio geográfico. De esa manera, tanto los dueños de empresa como los trabajadores pueden obtener ventajas de la aglomeración. Los primeros, al contar con una oferta amplia de trabajo especializado para la que resultan menores los costos de formación, en especial por un proceso de capacitación que se transfiere entre generaciones; los segundos, al reducir la dependencia de una única empresa demandante de trabajo.

Para finalizar, la proximidad geográfica ayuda a la propagación de la información, a través de flujos relacionados con las habilidades y conocimientos específicos de la industria, los cuales se difunden con facilidad entre empresas vecinas y dan lugar a un proceso acumulativo de saber hacer (know-how) propio de ella. Con esto 
ya Marshall está haciendo referencia al concepto de spillovers de conocimiento, al considerar la capacidad y la posibilidad de transferir ideas de un agente productivo a otro y convertirse en fuente de futuras innovaciones.

Marshall consideraba, entonces, que algunos sectores industriales podrían obtener ventajas de la producción a gran escala, a través de un proceso de reagrupamiento espacial que incluyera un número elevado de pequeños productores (conformación de un distrito industrial), y planteó de esa forma un modelo de crecimiento industrial alternativo a la gran empresa.

Partiendo de esa aproximación, Hoover (1937) establece una diferencia entre economías externas de localización y de urbanización. Las primeras derivan de la aglomeración de actividades de un sector productivo en particular y se consideran externas a las firmas que lo integran, pero internas a ese sector. Las economías de urbanización se originan en la concentración espacial de diversas actividades y sectores económicos, y resultan apropiables por la totalidad de empresas que comparten esa localización, es decir, son externas a un sector productivo determinado pero internas al espacio de análisis, como señala Camagni (2005). Entonces las economías externas de aglomeración son ventajas derivadas de un mayor tamaño de un sector productivo o grupo de empresas de diversos sectores, las mismas que se benefician de su proximidad en el espacio. De acuerdo con Duranton y Puga (2003) y Puga (2009), la microfundamentación de las mismas, parte de reconocer tres mecanismos básicos para su generación²: el favorecimiento de un entorno de aprendizaje a través de la interacción cara a cara (learning); la conformación de un mercado de trabajo amplio que facilita los emparejamientos (encuentros) -y la calidad de estos- entre empresas y trabajadores (matching); y la existencia de servicios intermedios y acceso compartido a bienes y servicios que presentan indivisibilidad para su suministro (sharing).

A partir de los trabajos de Scitovsky (1954) se plantea la distinción entre economías externas pecuniarias y tecnológicas, al diferenciar aquellas que derivan de los mecanismos de mercado de las que son una consecuencia de las interdependencias directas entre las unidades productivas. Estas últimas hacen presencia cuando el producto de una organización o firma empresarial $\left(\mathrm{y}_{1}\right)$, además de depender de sus factores de producción $\left(x_{1}, w_{1}, ..\right)$, guarda relación con el producto $\left(\mathrm{y}_{2}\right)$ y los factores productivos de otras empresas $\left(\mathrm{x}_{2}, \mathrm{w}_{2}, \ldots\right)$, dada la presencia de spillovers tecnológicos e informativos, tal como se expresa en la ecuación [1].

\footnotetext{
Otros trabajos en los que se aborda la discusión sobre estos mecanismos son Fujita, Krugman y Venables (1999), García y Muñiz (2010).
} 


$$
y_{1}=f\left(x_{1}, w_{1}, \ldots, y_{2}, x_{2}, w_{2}, \ldots\right)
$$

Las economías externas pecuniarias surgen a partir del beneficio $\left(b_{1}\right)$ de una organización o firma, el que a su vez depende de la cantidad producida y de los factores utilizados en su proceso productivo; además, al relacionarse con otras empresas en el mercado, dicho beneficio termina siendo afectado por la producción y los factores utilizados por otros productores como se expresa en la ecuación [2]. Estas economías se transmiten a través del sistema de precios y se generan porque la producción exterior creciente, sea del sector al que pertenece la empresa o de otros sectores, tiene por resultado variaciones en el precio del producto o de los insumos que requiere de la empresa.

$$
b_{1}=f\left(y_{1}, x_{1}, w_{1}, \ldots, y_{2}, x_{2}, w_{2}, \ldots\right)
$$

Por otra parte, Oughton y Whitam (1997) incorporan el concepto de economías externas colectivas, que se fundamentan en las ventajas derivadas de la cooperación interempresarial. Este tipo de externalidades tiene su origen cuando las empresas cooperan de manera concertada en determinadas actividades (como la investigación y el desarrollo tecnológico, la formación de los empleados, la promoción de los productos o el establecimiento de redes de exportación) para conseguir una disminución de los costos fijos ligados a las actividades que desarrollan. Las economías externas colectivas resultan así externas a las firmas empresariales que participan, pero internas al grupo integrante de la red de cooperación.

Desde el trabajo de Hoover (1937), las economías externas de aglomeración se concibieron estáticas en el tiempo y en el espacio. Contrario a ello, planteamientos posteriores, como los de Audretsch y Feldman (2004), destacan una dinámica temporal en estas economías y consideran que la historia del entorno económico es importante, ya que las empresas que actúan en él, se benefician de un stock de conocimiento acumulado y de unos servicios especializados desarrollados. Otros resaltan una dinámica espacial asociada a las interacciones que observan las ciudades; en ese sentido, dicha dinámica se puede entender cuando se asume la ciudad como un nodo en una red, antes que como una unidad aislada.

La perspectiva de la dinámica temporal de las economías externas, asocia estas con derrames de conocimiento y las divide de manera tradicional entre las denominadas (i) externalidades tipo MAR (Marshall, Arrow, Romer) definidas como fenómenos intraindustriales en contextos oligopolísticos; (ii) externalidades tipo Porter o intraindustriales con mercados competitivos y (iii) externalidades tipo Jacobs o interindustriales, también con mercados competitivos; de acuerdo con la tipología propuesta por Glaeser y otros (1992). 
La generación de economías externas espacialmente dinámicas es abordada por el paradigma de las redes de ciudades. Los factores de aglomeración que dan origen a las externalidades son intensos en las ciudades donde se concentra la población y la actividad económica, sin embargo, en los últimos años se ha puesto de manifiesto la posibilidad de que la interacción entre agentes económicos, individuos y organizaciones, localizados en distintas unidades urbanas, genere nuevas fuentes de economías externas, vía incrementos en el tamaño del mercado y posibles derrames de conocimiento. Ellas corresponden a economías que se generan no solo dentro de una ciudad, sino que se dan en la interacción que presentan los agentes económicos de un grupo de ciudades que conforman en su acción redes de ciudades. Como sugiere Soja (2008), desde el enfoque de redes de ciudades, se considera que no es necesaria la concentración espacial de las actividades para que surjan las externalidades, ya que estas pueden derivar de la interacción entre agentes, individuos y organizaciones y tener incidencia en el crecimiento de las ciudades comprometidas. En esa perspectiva, son economías externas a las unidades productivas y a las ciudades, pero internas a la red de ciudades que integran.

Capello (2000) percibe que las redes hacen posible que las ciudades pequeñas tengan las ventajas de aglomeración de una gran metrópoli y observa que las ciudades de tamaño intermedio se consideran cada vez más como los lugares que podrían albergar el crecimiento de los años venideros. Ciudades cuyo tamaño facilita el equilibrio medioambiental, la movilidad eficiente y la posibilidad de conservar un sentido de pertenencia en lo que respecta a la población. Si bien enfrentan debilidades frente a una metrópoli, referidas entre otras al tamaño del mercado y condiciones de centralidad, también, ello puede ser compensado por las interconexiones con otras ciudades cercanas a partir de las cuales se genera lo que nombra como urban

\section{network externalities.}

En el mismo sentido Parr (2002) sostiene que, si bien las economías de aglomeración se mencionan de manera frecuente con referencia al espacio intraurbano, otra escala que resulta relevante para esas economías es la regional. Advierte que cada vez es más reconocida la presencia de economías de aglomeración regional o externalidades regionales, términos que llaman la atención sobre el hecho de que las ventajas de la aglomeración pueden ser compartidas por las firmas empresariales en un ámbito regional, por lo que, para disfrutar de los beneficios de una metrópolis, ya no se requiere una localización en su interior, sino accesibilidad a ella.

En distintos trabajos Camagni, Capello y Caragliu $(2015 ; 2016)$ argumentan que, el reconocimiento de la presencia de ventajas derivadas de los procesos de aglomeración, no debe entenderse como una relación automática en la que un aumento del tamaño urbano conlleva de manera inexorable al crecimiento de la productividad y a 
la generación de rendimientos crecientes. Es decir, que el crecimiento demográfico de una ciudad no se traduce de manera automática en crecimiento económico, con lo que resultan múltiples los factores que contribuyen a la generación de ventajas de aglomeración, como las características demográficas y económicas del contexto y la capacidad para establecer la cooperación en red entre las unidades urbanas.

Entonces, una parte importante de las ventajas de aglomeración puede darse en la escala regional. Este tipo de externalidades se relaciona con lo que Robinson $(1948)^{3}$ denominó en su momento economías móviles, concepto con el cual quería resaltar que las empresas que se encuentran localizadas en espacios (ciudades) diferentes, pero articulados, cuentan con las mismas posibilidades de explotar ventajas respecto a aquellas localizadas en el mismo espacio y aún más, podrían disfrutar de menores costos frente a los que se derivan de una concentración espacial. Acá se identifican como economías externas de red, las cuales constituyen una preocupación reciente para la geografía económica.

\section{Tabla 1. Tipología de las economías externas de aglomeración}

\begin{tabular}{|c|c|c|c|}
\hline Ámbito & Estáticas & $\begin{array}{c}\text { Dinámicas (dimensión } \\
\text { temporal) }\end{array}$ & $\begin{array}{c}\text { Dinámicas (dimensión } \\
\text { espacial) }\end{array}$ \\
\hline Externas a la firma. & $\begin{array}{l}\text { Economías de locali- } \\
\text { zación. } \\
\text { Economías de urbani- } \\
\text { zación. }\end{array}$ & $\begin{array}{l}\text { Economías tipo MAR. } \\
\text { Economías tipo Por- } \\
\text { ter. } \\
\text { Economías tipo Ja- } \\
\text { cobs. }\end{array}$ & $\begin{array}{l}\text { Distrito industrial } \\
\text { marshalliano. } \\
\text { Economías colectivas. } \\
\text { Externalidades de red. }\end{array}$ \\
\hline $\begin{array}{l}\text { Internas al sector } \\
\text { (internas a la ciudad). }\end{array}$ & $\begin{array}{l}\text { Economías de Loca- } \\
\text { lización. }\end{array}$ & $\begin{array}{l}\text { Economías tipo MAR. } \\
\text { Economías tipo Por- } \\
\text { ter. }\end{array}$ & \\
\hline $\begin{array}{l}\text { Internas al sector } \\
\text { (externas a la ciudad). }\end{array}$ & & & $\begin{array}{l}\text { Distrito industrial } \\
\text { marshalliano. }\end{array}$ \\
\hline $\begin{array}{l}\text { Externas al sector } \\
\text { (internas a la ciudad). }\end{array}$ & $\begin{array}{l}\text { Economías de urbani- } \\
\text { zación. }\end{array}$ & $\begin{array}{l}\text { Economías tipo Ja- } \\
\text { cobs. }\end{array}$ & \\
\hline $\begin{array}{l}\text { Externas a la ciudad } \\
\text { (internas a la red de } \\
\text { ciudades). }\end{array}$ & & & $\begin{array}{l}\text { Distrito industrial } \\
\text { marshalliano } \\
\text { Economías colectivas. } \\
\text { Externalidades de red. }\end{array}$ \\
\hline
\end{tabular}

Fuente: elaborado con base a la bibliografía referenciada.

\footnotetext{
${ }^{3}$ El texto de Robinson fue escrito originalmente en 1931, con sucesivas reimpresiones.
} 
En la Tabla 1 se presenta una síntesis de las distintas fuentes de externalidades de aglomeración y se destaca el ámbito de la externalidad y su condición estática o dinámica. Las economías de red se definen como una externalidad para la firma empresarial, el sector económico y la unidad urbana, y pueden ser consideradas como internas a una red regional de ciudades. La geografía económica aborda el estudio de estas externalidades desde la perspectiva de la unidad urbana, e interpreta desde el paradigma de la red la organización de estas unidades las redes de ciudades.

\section{LOS ESTUDIOS RELACIONADOS CON LAS EXTERNALIDADES DE RED}

El trabajo empírico en torno a las redes y a la generación de externalidades es abordado en sus inicios por Capello (2000), que centró sus esfuerzos en estimar los impactos de una red urbana sobre el desempeño de las ciudades que la integran. Consideró los datos de encuestas aplicadas a las 36 ciudades europeas integrantes de la red de cooperación Healthy City Network administrada por la Organización Mundial de la Salud, regional europea ubicada en Copenhague. Esta información permitió construir indicadores complejos relacionados con las características estructurales de esas ciudades; objetivos y pactos; iniciativas, proyectos y acciones lanzadas gracias a la red y proyectos específicos en la red. A través de una estimación por mínimos cuadrados ordinarios (OLS, por sus siglas en inglés), encontró una relación positiva y significativa entre el desempeño de una ciudad, definido a través de la implementación de políticas urbanas exitosas, y el grado de conectividad que ella presenta con otras unidades urbanas, es decir, con la red de ciudades.

Trullen y Boix (2001) exploraron la capacidad de las ciudades para difundir el conocimiento como factor clave en el desarrollo económico en presencia de redes de ciudades. Su análisis se centró en la red regional de ciudades de Cataluña (España), asumieron el municipio como unidad de referencia, tomaron el empleo agregado en los sectores económicos de alto conocimiento como variable dependiente y, como una de las variables explicativas, el número de vínculos de red del municipio. A través de la estimación por OLS encontraron que la diversidad productiva de la entidad territorial y las relaciones de red explican de manera significativa el comportamiento del empleo en los sectores de alto conocimiento, siendo especial el caso de los municipios pequeños y medianos; lo que según los autores evidenció la presencia de externalidades de red originadas en los derrames de conocimiento que provocan las interacciones entre las ciudades.

En un trabajo posterior Trullen y Boix (2007) confrontaron la hipótesis según la cual el conocimiento conduce al crecimiento y ese conocimiento está relacionado con la existencia de externalidades de aglomeración y de red. Consideraron para 
esto último que la presencia de canales relacionales estables entre las ciudades, puede generar derrames de conocimiento y desde el paradigma de la red de ciudades asumieron que, contrario a lo que suponen los modelos de lugar central, la difusión del conocimiento no solo se puede realizar de manera vertical, sino también entre ciudades de igual rango. En el contexto intrarregional de Cataluña, contrastaron la existencia de economías de aglomeración dinámicas en la dimensión espacial (de red), a través de un modelo con especificaciones econométricas espaciales.

Otros trabajos destacables son los de Camagni, Capello y Caragliu (2015 y 2016) que se plantearon como tarea mostrar que las economías externas no están solo relacionadas con el tamaño de la ciudad, por el contrario, algunas características urbanas específicas como la presencia de funciones de alto nivel y las redes de cooperación entre las ciudades, potencian los rendimientos crecientes. Los resultados alcanzados sugirieron que las ciudades pueden lograr economías de aglomeración a pesar de un tamaño urbano limitado.

Pero un ejercicio de investigación bastante cercano a los propósitos de este trabajo es el de Boix (2003). En el mismo se propuso avanzar en el estudio de la relación entre las redes de ciudades y la generación de economías externas que afectan al crecimiento y al desarrollo económico; para lo cual se estimaron los efectos de las economías de concentración (aglomeración) y de red a través de un modelo econométrico mixto regresivo-regresivo espacial, el cual incluyó como variable dependiente un indicador de crecimiento del municipio, la tasa de variación de la ocupación; y como explicativas, un conjunto de variables de los demás municipios relacionadas con número de ocupados, número de empresas y población. Se observó una relación causal entre la organización de las unidades urbanas que forman redes de ciudades y la generación de economías externas que afectan al crecimiento y desarrollo económico, por lo que estas economías deben ser consideradas como fuente adicional de externalidades en la producción.

En general, los trabajos se diferencian en distintos aspectos, como la naturaleza de los datos y las variables incluidas en la estimación de los modelos, sin embargo, todos ellos coinciden en utilizar datos agregados por municipio. Con excepción de Capello (2000), que orienta su investigación a observar la presencia de externalidades en una red de cooperación, los distintos trabajos identifican redes regionales de ciudades y exploran en ellas la presencia de economías de red. De otro lado, en el modelado que apoyó las estimaciones, hacen presencia ejercicios más simples que se apoyan en regresiones de correlación sin clara fundamentación teórica, como en Capello (2000), y aproximaciones que parten de modelos teóricos de base económica como en Trullen y Boix (2001; 2007), Boix (2003), Camagni, Capello y 
Caragliu $(2015 ; 2016)$.

\section{ASPECTOS METODOLÓGICOS}

Resultan tradicionales dos caminos en la obtención y tratamiento de los datos, al igual que dos formas de aproximación para la medición de las economías de concentración (aglomeración) y de red. En cuanto a lo primero, los datos pueden ser tomados para la firma empresarial o de manera agregada para una ciudad o municipio, dependiendo de la disponibilidad de información y en relación con lo que se quiere contrastar; es decir, el impacto de estas externalidades sobre la producción en el caso de agentes individuales (firmas) o de manera agregada para los municipios, como es el caso de este estudio. En cuanto a lo segundo, las formas de modelar la medición de las economías de aglomeración y de red, según describe Boix (2003), algunas aproximaciones consideran la realización de regresiones de correlación que intentan relacionar una variable representativa del crecimiento económico del municipio con una serie de variables que se considera expresan condiciones de concentración o relaciones de red con otros municipios, según sea el caso. Otras parten de un modelo teórico de base económica que no requiere estar especificado a partir de una función de producción y tiene como propósito observar los factores explicativos de las dinámicas de crecimiento del municipio ; pero en sus distintas opciones se desarrolla un trabajo empírico similar que con sidera una ecuación de regresión, en la que se incluyen coeficientes que recogen los efectos de economías externas.

Las regresiones de correlación constituyen lamaproximación más simple, dado que en lo fundamental están orientadas a establecer asociación entre la variable dependiente y las variables explicativas y no tienen que estar soportadas por un modelo teórico definido. Por ello mismo, su ventaja está en la sencillez de la especificación, a lo que se suma que, en ocasiones, la falta de datos hace de este ejercicio la única aproximación posible.

En este trabajo se asume un modelo de regresión no basado en funciones de producción para explorar la existencia de economías de red en el sistema urbano ciudad-región Eje cafetero, integrado por 15 municipios de cuatro departamentos del centro occidente colombiano, Manizales, Pereira, Armenia, Neira, Palestina, Villamaría, Chinchiná, Santa Rosa de Cabal, Dosquebradas, La Virginia, Cartago, Circasia, Calarcá, Montenegro y La Tebaida; en el cual Calvo y Gaviria (2014) evidenciaron la presencia de una red de ciudades. Se parte de considerar que el crecimiento de un municipio $\left(\mathrm{y}_{\mathrm{i}}\right)$ está influido por la dinámica económica de los otros municipios $\left(\mathrm{y}_{\mathrm{j}}\right)$ de su red, así como por otras características de estos municipios, sin embargo, dadas 
las limitaciones de información que hacen improbable contar a escala municipal con series de variables distintas a aquellas que aproximan su crecimiento económico, en el modelo a estimar solo es posible incluir la variable referida al crecimiento de otros municipios. De esta manera, el ejercicio sugerido exige la estimación de una ecuación de regresión de la forma,

$$
y_{i}=\alpha+\beta y_{j}+\varepsilon ; i \neq j
$$

Con $\varepsilon$ : término de error.

Dada la posible presencia de autocorrelación o dependencia espacial, se consideró un análisis exploratorio previo apoyado en estadísticos de contrastación como el índice de Moran (I), para lo cual se utilizó como matriz de retardos o de contactos $\mathrm{W}$, a la matriz binaria de contigüidad espacial, que se construye asignándole 1 a las observaciones vecinas al punto y cero a las demás observaciones. En caso de evidenciarse dependencia espacial, acogiendo los procedimientos sugeridos por la econometría espacial para estos casos, se estima como alternativa un modelo de retardo espacial de la forma,

$$
y_{i}=\alpha+\delta \mathrm{W} y_{i}+\beta y_{j}+\varepsilon ; i \neq j
$$

En donde $\mathrm{y}_{\mathrm{i}}$ es un vector ( $\mathrm{Nx} 1$ ), con $\mathrm{N}$ número de observaciones; W es la matriz de retardos o contactos $(\mathrm{NxN})$; $\mathrm{y} \mathrm{Wy}$ es el operador de retardo espacial de la variable $\mathrm{y}_{\mathrm{i}}$, la variable dependiente en el modelo.

Dado que en Colombia no se dispone de cuentas económicas a escala municipal, resultó necesario recurrir al uso de las captaciones bancarias como una variable proxy del Producto Interno Bruto (PIB) o ingreso municipal, información suministrada por la Superintendencia Financiera de Colombia y disponible en datos trimestrales, a escala municipal y desde 1996. Otros estudios como los de Bonet y Meisel (1999), Galvis (2001) y Barón (2002) han recurrido a este indicador como proxy del crecimiento de los ingresos, apoyados en la existencia de correlaciones positivas entre ambas variables. En este sentido, Bonet y Meisel (1999) utilizan datos para los departamentos colombianos y observan que en el año 1960 la correlación entre las dos variables fue de 0,83 y en 1950 de 0,77. De manera adicional, realizaron estimaciones para los años 1960 y 1995 en las que el coeficiente varió de 0,78 a 0,88. Por su parte, Barón (2002) realizó otras estimaciones similares en las que encontró correlaciones aceptables. En el caso del Eje Cafetero, se estimaron coeficientes de correlación positivos y significativos de 0,79, 0,87 y 0,93 para los departamentos de Quindío, Risaralda y Caldas, en el período 1996-2013. 


\section{ECONOMÍAS EXTERNAS DE RED EN LA CIUDAD REGIÓN}

Como se señaló antes, el propósito es estimar a escala municipal la existencia de dichas externalidades en el sistema urbano ciudad-región Eje Cafetero. Para ello se exploró evidencia sobre la presencia de relaciones entre el desempeño económico de los distintos municipios que la integran, utilizando como proxy los datos sobre captaciones bancarias, información trimestral entre 1996 y 2014.

Para explorar la presencia de autocorrelación o dependencia espacial en el comportamiento de las captaciones bancarias, a través del índice de Morán (IM), se realizó una prueba de hipótesis que buscó evidencia estadística que aclare si la configuración espacial de la variable se produce de manera aleatoria. Para ello se definió como hipótesis nula que la configuración espacial de las captaciones bancarias se produce de manera aleatoria y como hipótesis alternativa que esa configuración no se produce de manera aleatoria.

Mediante el software Stata se estimó un índice de Morán I igual a -0,00186234, cuyo valor estandarizado correspondiente es $Z(I)=-0,1527524$, con un $z$-value de $-0,1680$ (gráfica 1), por lo que resulta un valor no significativo que no permite rechazar la hipótesis nula y ofrece evidencia de la no presencia de autocorrelación espacial en el comportamiento de las captaciones bancarias. En igual sentido, en Calvo (2015) se estimó el índice de Morán I para la serie tasa de crecimiento de las captaciones bancarias en los municipios de la ciudad región, durante el período 1996-2014, encontrándose un valor $\mathbf{I}=-0,062$ que de igual manera evidenció ausencia de autocorrelación espacial en dicha variable.

Gráfica 1. Diagrama de dispersión de Moran para las captaciones bancarias

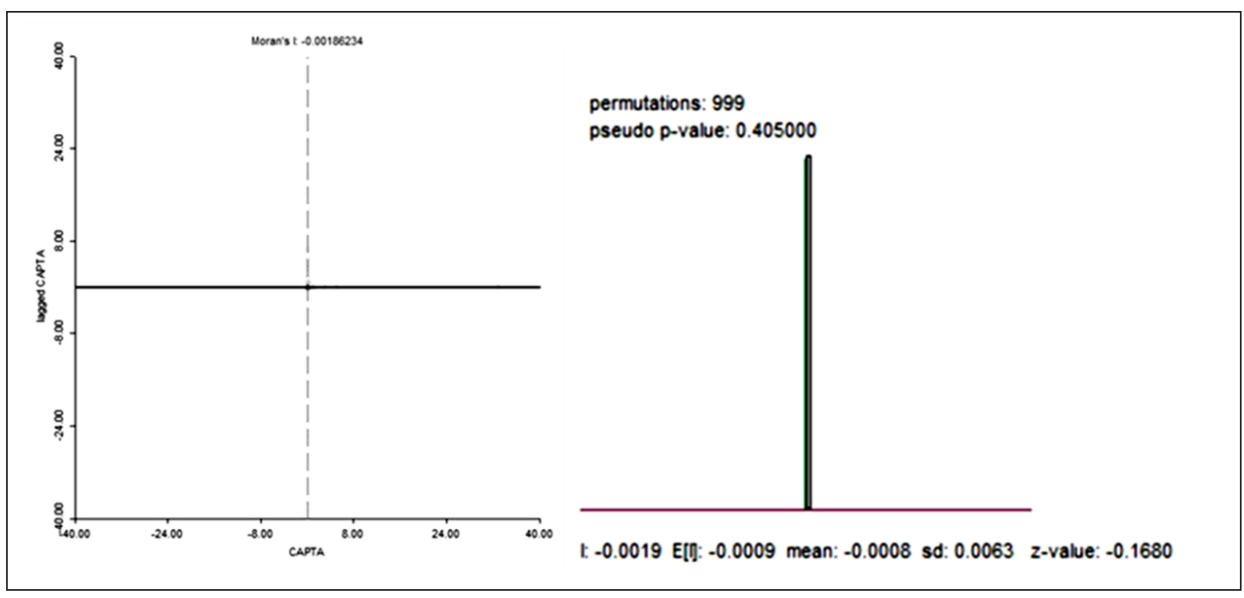

Fuente: cálculos con base en información de la Superintendencia Financiera de Colombia, 2014. 
Dada la evidencia de no autocorrelación espacial se recurrió a la econometría tradicional. A través de ella es posible analizar un conjunto de datos de diferentes formas, como es el caso de una regresión lineal, en la cual no se diferencian los individuos o unidades de estudio que participan (familias, empresas, sectores, ciudades, entre otros), es decir, se tratan como parte de un pool de datos (regresión agrupada) o, de otra forma, se pueden tener en cuenta los efectos individuales cuando estos existen, como es el caso de las técnicas de panel (regresión de datos anidados).

Los paneles de datos son bases de información que contienen elementos de corte transversal (los individuos), cuya evolución puede ser observada en el tiempo, como ocurre en el caso del presente trabajo, al contar con información sobre las captaciones bancarias en los municipios de la ciudad-región (individuos o información de corte transversal, en la técnica de panel) y su evolución trimestral entre 1996 y 2014. Para optar por desarrollar un análisis de panel de datos se debió realizar, en primera instancia, una verificación de que la información permitía descontar efectos individuales y de esa manera era conveniente tratarla como un panel de datos. Es decir, si una muestra presenta efectos individuales $\left(\propto_{i}\right)$ y las variaciones en el tiempo también explican el comportamiento de las variables, entonces se debe aplicar esta metodología; si por el contrario no se observa este tipo de condición, no existen efectos individuales $(\propto)$, entonces una estimación tipo pool que OLS sería consistente y eficiente.

Si se encuentra evidencia de efectos individuales, en una segunda instancia, es necesario definir si esos efectos individuales $\left(\alpha_{i}\right)$ deben ser tratados como aleatorios o fijos, es decir, se debieron realizar pruebas que definieran el modelo más apropiado entre efectos fijos (FE) o efectos aleatorios (RE). Una síntesis de esta línea metodológica se muestra en la gráfica 2, donde $\mathbf{i}, \mathbf{j}$ se refieren a los individuos o unidad de estudio (corte transversal) y $\mathbf{t}$ a la dimensión en el tiempo.

\section{Gráfica 2. Algoritmo para la especificación del modelo de regresión}

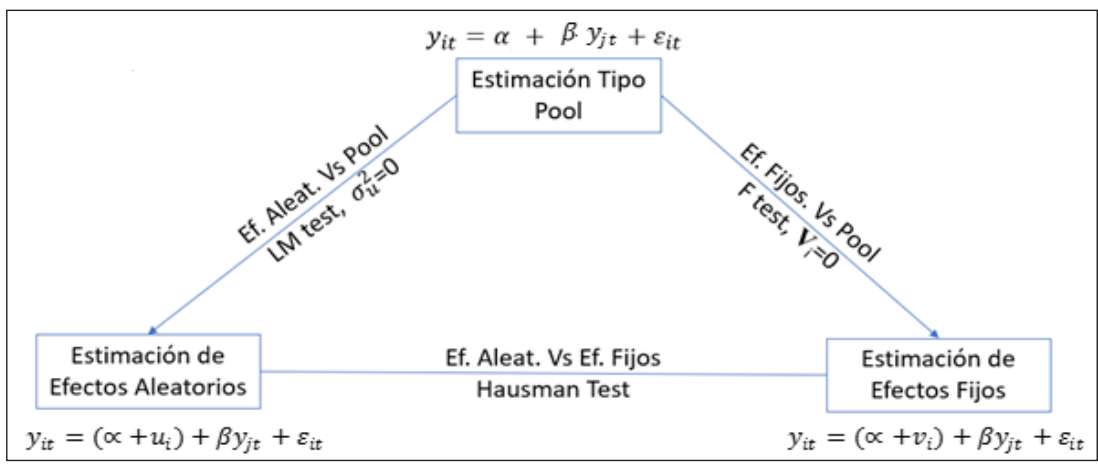

Fuente: elaborado con base en Moreno y Vayá (2000). 
De acuerdo con este algoritmo, el proceso de implementación de un modelo de datos de panel inició con una estimación tipo pool, en la que no se diferencian los individuos o unidades de estudio (municipios) en tanto se asumen como una observación más en un momento del tiempo, por ello, en la ecuación a estimar se supone que el intercepto de la regresión ( $\propto$ ) es el mismo para todos los municipios.

Frente a los resultados que ofrecen las estimaciones de las ecuaciones tipo pool y efectos aleatorios, si se cumple que la varianza de u es cero $\left(\sigma_{u}^{2}\right.$ $=0$ ), se puede concluir que no existe diferencia entre los dos modelos. Entonces, para discernir entre cuál de los modelos es el adecuado, se aplica la prueba del multiplicador de Lagrange para efectos aleatorios (RE) teniendo presente la hipótesis nula Ho: $\mathrm{O}_{\mathrm{u}}^{2}=0$ y una Chi-cuadrado de contraste. Si se rechaza Ho, se concluye que los modelos son diferentes luego, es preferible recurrir a la estimación de efectos aleatorios, que incorpora en el análisis las diferencias entre los municipios desde las interacciones. Dados los resultados que se presentan en la tabla 2 no se rechaza la Ho, de lo que se deriva que es indiferente utilizar una estimación tipo pool o efectos aleatorios.

Tabla 2. Pruebas de datos de panel

\begin{tabular}{|c|c|c|c|c|c|}
\hline $\begin{array}{c}\text { Pool vs. RE } \\
\text { LM-test } \\
p \text {-value }\end{array}$ & Hipótesis & $\begin{array}{c}\text { Pool vs. FE } \\
\text { F-test } \\
p \text {-value }\end{array}$ & Hipótesis & $\begin{array}{c}\text { RE vs. FE } \\
\text { Hausman } \\
\text { p-value }\end{array}$ & Hipótesis \\
\hline 0,999 & $\begin{array}{l}\text { No hay dife- } \\
\text { rencia siste- } \\
\text { mática entre } \\
\text { pool y RE. }\end{array}$ & 0,0000 & $\begin{array}{l}\text { Existe diferen- } \\
\text { cia sistemática } \\
\text { entre pool y FE. }\end{array}$ & 0,0000 & $\begin{array}{l}\text { Existen diferencias sistemá- } \\
\text { ticas entre los coeficientes } \beta \\
\text { estimados por efectos fijos y } \\
\text { efectos aleatorios. }\end{array}$ \\
\hline
\end{tabular}

Fuente: cálculos en Stata con base en información de la Superintendencia Financiera de Colombia.

Sin embargo, se debió realizar un ejercicio similar entre el modelo tipo pool y el de efectos fijos. Para ello se recurre al test F de los efectos individuales, el mismo que, de acuerdo con los resultados que aparecen en la tabla 2, permite rechazar la hipótesis nula en la que se plantea que el componente de los efectos individuales $v_{i}$ es igual a 0 (Prob $>F=0,000$ ), lo que justifica un análisis que considere los efectos individuales.

Al final, al considerar esta última evidencia de presencia de efectos individuales y entendiendo que estos pueden ser tratados como aleatorios o fijos, fue necesario evaluar si existe diferencia sistemática entre un modelo de efectos fijos (FE) y un modelo de efectos aleatorios (RE); teniendo en cuenta que el modelo FE asume que existe un término constante diferente para cada individuo y supone que los efectos 
individuales son independientes entre sí, en tanto que el modelo RE considera que los efectos individuales no son independientes entre sí y están distribuidos de manera aleatoria alrededor de un valor dado, siendo " $\mathrm{u}_{\mathrm{i}}$ " la perturbación aleatoria que permite distinguir el efecto de cada individuo en el panel.

Para decidir cuál es el estimador más adecuado para el modelo de panel (fijo o variable) se empleó el Test de Hausman, un test que compara los coeficientes $\beta$ obtenidos por medio del estimador de efectos fijos y efectos aleatorios e identifica si las diferencias entre ellos son o no significativas. El mismo utiliza una prueba Chi-cuadrado cuyo estadístico se calcula a partir de las diferencias entre los $\beta$ ponderados por la varianza; la hipótesis nula plantea que no existe diferencia sistemática entre los coeficientes. Si se acepta Ho, ello sugiere que los estimadores RE y FE no presentan diferencias sistemáticas, luego es más conveniente utilizar efectos aleatorios, un estimador eficiente y consistente; en caso contrario es preferible elegir el modelo de efectos fijos, un método menos eficiente (la varianza de la estimación es mayor) pero más consistente (el valor del parámetro estimado es menos sesgado).

Dados los resultados contenidos en la tabla 2, un $\mathrm{p}$-value $<0,05$ permite rechazar la hipótesis nula por lo que se hace conveniente utilizar el estimador de efectos fijos. Con esa evidencia se realizaron las estimaciones de efectos fijos para cada uno de los municipios de la ciudad región (tabla 3), obteniendo valores de los efectos individuales positivos y significativos que indican la existencia de interacción entre los municipios, una interacción que se revela con efectos positivos dado el valor estimado de la pendiente de la relación de 0,519833 , significativo al $1 \%$.

De esta manera, a través del ejercicio de panel de efectos fijos se obtuvo evidencia que apoya la hipótesis de presencia en la ciudad-región de externalidades dinámicas en la dimensión espacial, derivadas de la interacción en red de los municipios que la integran, esto dado el efecto positivo del desempeño económico de esos municipios sobre el conjunto de la ciudad región, que deriva de las relaciones existentes y se manifiesta a través de los signos y la significancia estadística de los coeficientes que corresponden a los efectos individuales de los municipios y la pendiente de la regresión. Dicho efecto positivo podría estar relacionado con factores sectoriales, derivados de una estructura productiva de los municipios en gran medida dependiente del sector cafetero, antes que ser fruto de la presencia de ventajas de aglomeración con origen en los vínculos en red que ellos sostienen. Esa posibilidad se descarta en tanto el nuevo mapa cafetero de Colombia muestra que los departamentos de Caldas, Quindío y Risaralda han dejado de ser el corazón de esa actividad (DANE, 2015) y los centros principales de la ciudad-región observan una importante diversificación productiva con amplia presencia de actividades terciarias. 
Tabla 3. Efectos individuales de interacción en los municipios de la ciudad región

\begin{tabular}{|c|c|}
\hline \multicolumn{2}{|c|}{$\begin{array}{l}\text { Cross-sectional time-series FGLS regression } \\
\text { Coefficients: generalized least squares } \\
\text { Panels: heteroskedastic with cross-sectional correlation } \\
\text { Correlation: common AR(1) coefficient for all panels }(-0.0380) \\
\text { Estimated covariances }=120 \text { Number of obs }=1,095 \\
\text { Estimated autocorrelations }=1 \text { Number of groups }=15 \\
\text { Estimated coefficients }=16 \text { Time periods }=73 \\
\text { Wald chi2 }(15)=4126,41 \\
\text { Prob }>\text { chi } 2=0,0000\end{array}$} \\
\hline Municipio & Coef. Std. Err. $z$ P $>|z|[95 \%$ Conf. Interval $]$ \\
\hline Armenia & $244,24281,7324711,240,000201,6479286,8377$ \\
\hline Calarcá & $23,307241,94346211,990,00019,4981227,11635$ \\
\hline Cartago & $67,983545,61756212,100,00056,9733378,99376$ \\
\hline Chinchiná & $18,928841,6946211,170,00015,6074522,25024$ \\
\hline Circasia & $1,9781390,186421510,610,0001,612762,34351$ \\
\hline Dosquebradas & $31,549513,4052279,270,00024,8753838,22363$ \\
\hline La Tebaida & $3,5695050,353347310,100,0002,8769574,262053$ \\
\hline La Virginia & $9,6951710,840486611,540,0008,04784711,34249$ \\
\hline Manizales & $423,852937,2664711,370,000350,812496,8939$ \\
\hline Montenegro & $7,709330,632504812,190,0006,4696448,949017$ \\
\hline Neira & $4,5901850,453038210,130,0003,7022465,478124$ \\
\hline Palestina & $1,7791930,18844989,440,0001,4098382,148548$ \\
\hline Pereira & $483,440346,028910,500,000393,2253573,6553$ \\
\hline $\begin{array}{l}\text { Santa Rosa de } \\
\text { Cabal }\end{array}$ & $21,425171,88167311,390,00017,7371625,11318$ \\
\hline Villamaría & $4,1536070,407134810,200,0003,3556384,951577$ \\
\hline Pendiente & $0,5198330,026982519,270,0000,46694820,5727178$ \\
\hline
\end{tabular}

Fuente: cálculos en Stata con base en información de la Superintendencia Financiera de Colombia.

Al considerar el valor absoluto de los coeficientes de efectos individuales, en su orden los centros principales: Pereira, Manizales y Armenia, se muestran como los de mayor incidencia en el desempeño conjunto de los municipios de la ciudad región. Otros municipios que muestran efectos individuales de importancia destacada son Cartago, Dosquebradas, Calarcá, Santa Rosa de Cabal y Chinchiná. En general, los municipios de ese sistema de ciudades con superiores condiciones de centralidad y accesibilidad, según Gaviria (2018), son a su vez los que observan mayores efectos individuales sobre el conjunto de los municipios de la ciudad-región. En el caso de los conglomerados, el de Pereira y sus municipios aledaños, se muestra como 
el de mayores efectos individuales en el desempeño conjunto de la ciudad-región, el mismo que de manera coincidente se presenta como el de mayor cohesión en la red de ciudades, menor grado de concentración de funcionalidades y actividad económica en la ciudad capital y principal fuente de generación de valor agregado en la ciudad-región, como se muestra en Gaviria (2017).

Entre los municipios conurbados a su capital, Villamaría es el que evidencia menor grado efectos individuales, lo cual coincide con otras características observadas en esta entidad territorial, como su bajo nivel de centralidad en la red de ciudades y su discreto desarrollo funcional y económico. Características estas que lo colocan en desventaja para aprovechar las externalidades que se desprenden de la red de ciudades a la que pertenece. Entonces, al igual que Boix (2003), Capello (2000), Camagni, Capello y Caragliu (2015; 2016), Trullen y Boix (2001; 2007), el ejercicio realizado suma evidencia sobre la generación de externalidades a partir de las interacciones espaciales que sostienen las entidades territoriales. Según la misma, para que se produzcan economías externas de orden espacial, no es necesario que las unidades económicas y la población estén concentradas en una misma ciudad, como lo supone la idea tradicional de economías de aglomeración, y en ese sentido, no es indispensable el crecimiento y la expansión de una ciudad o municipio para acceder a dichas economías, aquellos municipios de menor tamaño pueden beneficiarse de las externalidades dinámicas en el espacio a partir de la relación que establezcan con su centro principal y otros municipios cercanos.

A diferencia de los trabajos anteriores, en este se desarrolló un ejercicio de panel en el que se hizo análisis de información que contenía elementos de corte transversal (los municipios), cuya evolución pudo ser observada por trimestres, entre 1996 y 2014; procedimiento a través del cual se logró tener en cuenta los efectos individuales de cada municipio sobre el desempeño económico de la ciudad región, este último observado a través del indicador proxy captaciones del sistema bancario en los distintos municipios.

\section{CONCLUSIONES}

De acuerdo con las evidencias encontradas en este trabajo, las redes hacen posible que las ciudades pequeñas tengan las ventajas de aglomeración de una gran metrópoli, haciendo plausible que ciudades de tamaño intermedio, como lo son las tres capitales de la ciudad-región, alberguen de manera gradual el crecimiento de los años venideros e impulsen con mayor fuerza el desarrollo de sus municipios aledaños. Ciudades cuyo tamaño contribuye a reducir los costos de la aglomeración, facilita el equilibrio medioambiental, favorece la movilidad eficiente y eleva la posibilidad 
de conservar un sentido de pertenencia en lo que respecta a la población. Si bien pueden ver reducidas las ventajas de la concentración, referidas al tamaño del mercado laboral y de bienes y servicios, por otro lado, ello puede ser compensado por las interacciones con otras ciudades cercanas.

Por lo mismo, si la urgencia de las grandes metrópolis es volverse competitivas y manejar problemas de escala, la necesidad de las ciudades intermedias y de los pequeños poblados pasa en principio por articularse a los centros urbanos vecinos y desarrollar sinergias económicas, sociales y políticas que les permitan disfrutar de las ventajas de la aglomeración a escala regional sin tener que asumir, al menos de manera plena, los costos de dicha concentración de actividad económica y población.

En el caso de la ciudad-región Eje Cafetero se hace fundamental el fortalecimiento de la cohesión territorial, para lo cual son pertinentes los esquemas de asociatividad territorial en la línea propuesta por la Ley 1454 de 2011, Orgánica de Ordenamiento Territorial (LOOT). A través de dichas dinámicas asociativas municipales se potencia la implementación de procesos de planificación más profundos que le apuesten a la gobernanza y al modelo suprarregional. De esa manera se avanza en el desarrollo de proyectos de interés regional que favorecen una mayor cohesión de la red, como es el caso de aquellos relacionados con los sistemas integrados de transporte interurbano planteados desde el gobierno nacional y desde el sector académico (tren-tranvía de cercanías y sistema de transporte intermunicipal -SITEJE-), destacados en Caracol Radio (2016), Duque (2016) y Meneses, Giraldo y Escobar (2009).

La propuesta del gobierno nacional en materia de transporte público interdepartamental cobija a los municipios de Pereira, Santa Rosa de Cabal, Manizales, Villamaría, Chinchiná, Armenia, Circasia, Calarcá, La Tebaida y Cartago. Por su parte, el tren-tranvía de cercanías se plantea a partir de la recuperación de la banca del antiguo ferrocarril y considera un recorrido en tren desde Villamaría hasta Dosquebradas y desde Pereira a Cartago; al entrar a Manizales y Pereira, pasaría a un funcionamiento en modo tranvía para transitar como transporte colectivo por las avenidas de la espina dorsal urbana en ambas ciudades.

La iniciativa SITEJE fue presentada en el Consejo Comunal 234 realizado durante el gobierno de Álvaro Uribe Vélez, y la misma propone integrar los sistemas de transporte urbano de pasajeros de Manizales, Pereira, Armenia y Cartago con el transporte interurbano entre esas ciudades. La propuesta plantea como propósitos la ampliación del mercado, la cohesión del territorio y la eficiencia en la prestación del servicio público de transporte de pasajeros, en coherencia con la idea de favorecer y aprovechar las externalidades que derivan de la interacción en una red de ciudades. 


\section{BIBLIOGRAFÍA}

Audretsch, David y Feldman, Maryann (2004). Knowledge Spillovers and the Geography of Innovation. En: Vernon Henderson, Gilles Duranton y William Strange (Eds.). Handbook of Urban and Regional Economics, Amsterdam, Holanda: North Holland, vol. 4, p. 2713-2739.

Barón, David (2002). Las regiones económicas de Colombia: un análisis de clusters. En: Documentos de trabajo sobre económica regional, n. ${ }^{\circ} 23$, p. 3-37.

Boix, Rafael (2003). Redes de ciudades y externalidades. Tesis doctoral. Departamento de Economía Aplicada, Universitat Autónoma de Barcelona, España, 360 p.

Bonet, Jaime y Meisel, Adolfo (1999). La convergencia regional en Colombia: una visión de largo plazo, 1926-1995. En: Documento de trabajo sobre economía regional, n. ${ }^{\circ}$, p. 1-50.

Calvo, Johana (2015). Entornos de red en la ciudad-región Eje Cafetero y generación de externalidades. Informe de investigación. Pereira, Colombia: Colciencias y Universidad Católica de Pereira, $110 \mathrm{p}$.

Camagni, Roberto; Capello, R0berta y Caragliu, Andrea (2015). Agglomeration economies in large versus small cities: similar laws, high specifities. En: Kourtit, Karima; Nijkamp, Peter y Stough, Roger (Eds.), The Rise of the City Spatial Dynamics in the Urban Century. New Horizons in Regional Science Series. Cheltenham, Reino Unido: Edward Elgar Publishing, p. 85-114.

Camagni, Roberto; Capello, Roberta y Caragliu, Andrea (2016). Static vs. dynamic agglomeration economies. Spatial context and structural evolution behind urban growth. En: Papers in Regional Science, vol. 95, n. ${ }^{\circ}$ 1, p. 133-158.

Camagni, Roberto (2005). Economía urbana. Barcelona, España: Bosch, 299 p.

Calvo, Johana y Gaviria, Mario (2014). La red de ciudades en la ciudad-región Eje Cafetero. En: Gestión y Región, n. ${ }^{18}$, julio-diciembre, p. 25-47.

Capello, Roberta (2000). The City Network Paradigm: Measuring urban network externalities. En: Urban Studies, vol. 37, n. ${ }^{\circ} 11$, p. 1925-1945.

Caracol Radio (2016). Sistema Integrado de Transporte se implementaría para eje cafetero y norte del Valle. Armenia, Colombia.

DANE $\square$ Departamento Nacional de Estadística $\square$ (2015). Censo nacional agropecuario, décima entrega de resultados, 2014 (tomo 2). Bogotá, Colombia: DANE, 1036 p.

Duque, Gonzalo (2016). Transporte integrado para la ciudad región. Bogotá, Colombia: Universidad Nacional de Colombia, p. 1-2.

Duranton, G. y Puga, Diego (2003). Micro-fundations of urban agglomeration economies. En: NBER Working Paper series, n. ${ }^{\circ}$ 9931, 61 p.

Fujita, Masahisa; Krugman, Paul y Venables, Anthony (1999). The spatial economy. Cities, regions and internacional trade. London, Reino Unido: The MIT press, $357 \mathrm{p}$.

Galvis, Armando (2001) Topografía económica de Colombia. En: Documentos de trabajo sobre economía regional, n. 22 , p. 1-45. 
García, Miguel y Muñiz, Iván (2010). El impacto espacial de las economías de aglomeración y su efecto sobre la estructura urbana. El caso de la industria de Barcelona 1986-1996. Barcelona, España: Universidad Autónoma de Barcelona, 30p.

Gaviria, Mario (2018). Redes y territorialidad en la ciudad-región Eje cafetero. En: Desenvolvimiento, Fronteras e Ciudadanía, vol. 2, n. ${ }^{\circ}$ 1, p. 75-79.

Gaviria, Mario (2017). Configuración espacial de la formación urbana regional del Eje Cafetero colombiano. En: Cuadernos de Geografía: Revista Colombiana de Geografía, vol. 26, n. ${ }^{\circ}$ 1, p.155-170.

Glaeser, Edward; Kallal, Hedi.; Scheinkman, José. y Shleifer, Andrei (1992). Growth in Cities. En: Journal of Political Economy, vol. 100, n. ${ }^{\circ}$ 6, p. 1126-1152.

Hoover, Edgar (1937). Location Theory and the Shoe and Leather Industries. Cambridge, Reino Unido: Harvard University Press, 323 p.

Marshall. Alfred (2005). Principios de economía. Madrid, España: Síntesis,1072 p.

Meneses, Hernán; Giraldo, Tania y Escobar, Diego (2009). Sistema integrado de transporte regional del Eje Cafetero Si-teje. Manizales, Colombia: Instituto de Estudios Urbanos, Universidad Nacional, $21 \mathrm{p}$.

Moreno, Rosina y Vayá, Esther (2000). Técnicas econométricas para el tratamiento de los datos espaciales. Barcelona, España: Ediciones Universitat de Barcelona, 158 p.

Oughton, Christine y Whittam, Geoff (1997). Competition and cooperation in the small firm sector. En: Scottish Journal of Political Economy, vol. 44, n. ${ }^{\circ}$ 1, febrero, p. 1-31.

Parr, John (2002). Agglomeration economies: ambiguities and confusions. En: Environment and Planning, n. 34 , abril, p. 717-731.

Puga, Diego (2009). The magnitude and causes of agglomerations economies. Madrid, España: IMDEA Social Sciences and CEPR, 19 p.

Robinson, Edward (1948). The structure of competitive industry. Londres, Reino Unido: Cambridge University Press, 156 p.

Scitovsky, Tibor (1954). Two concepts of external economies. En: The Journal of Political Economy, vol. 62, n. $^{\circ}$, abril, p. 143-151.

Soja, Edward (2008). Postmetrópolis. Estudios críticos sobre las ciudades y las regiones. Madrid, España: Traficantes de Sueños, 590 p.

Trullén, Jean y Boix, Rafael (2007). Knowledge, networks of cities and growth in regional urban systems. En: Papers in Regional Science, vol. 86, n. ${ }^{\circ}$ 4, noviembre, p. 551-574.

Trullén, Jean y Boix, Rafael (2001). Economia della conoscenza e reti di città: città creative nell'era della conoscenza. En: Sviluppo Locale, vol. 8, n. ${ }^{\circ} 18$, p. 1-21. 